\title{
Quantum key distribution without the wavefunction
}

\author{
Gerd Niestegge \\ Zillertalstrasse 39, 81373 München, Germany \\ gerd.niestegge@web.de
}

\begin{abstract}
A well-known feature of quantum mechanics is the secure exchange of secret bit strings which can then be used as keys to encrypt messages transmitted over any classical communication channel. It is demonstrated that this quantum key distribution allows a much more general and abstract access than commonly thought. The results include some generalizations for the Hilbert space version of quantum key distribution, but base upon a general non-classical extension of conditional probability. A special state-independent conditional probability is identified as origin of the superior security of quantum key distribution and may have more profound implications for the foundations and interpretation of quantum mechanics, quantum information theory, and the philosophical question what actually constitutes physical reality.
\end{abstract}

Key Words: Quantum key distribution, No-cloning theorem, Foundations of quantum theory, Quantum logic

PACS: 03.67.Dd, 03.65.Ta

\section{Introduction}

In the past thirty years, quantum cryptography has been a large field of extensive theoretical and experimental research. Several quantum key distribution protocols have been elaborated, their technical feasibility has been discussed, experimental set-ups and some practical implementations have been realized. Quantum key distribution may be the first commercial application of quantum information theory. A vast amount of papers on these topics are available by now (Each of the two pioneering works [7] and [18] has more than ten thousand and almost eight thousand quotations, respectively; for a review, see [20] or [37). The present paper, however, focuses on the theoretical foundations and concentrates on the two best-known quantum key distribution protocols.

Quantum mechanics renders possible the secure exchange of secret bit strings which can then be used as keys to encrypt messages transmitted over a classical communication channel. The quantum no-cloning theorem [16, 43, 45] ensures that an eavesdropper cannot steal the key without being detected. These results follow from the usual quantum mechanical Hilbert space formalism. After it could recently be shown in Ref. 32 that the quantum no-cloning theorem can be extended to a much more general and abstract framework - the quantum 
logics which possess a conditional probability calculus -, a similar extension of quantum key distribution shall be presented in this paper.

A quantum logic $E$ is a purely algebraic structure. It is quite common to use an orthomodular partially ordered set or lattice [5, 9, 25, 34. Its elements can be interpreted in different ways: as events, propositions or properties of the quantum system under consideration. This is why they are called propositions by some authors and events, preferably in probabilistic approaches, by others.

States are then defined in the same way as the classical probability measures, and conditional probabilities are postulated to behave like the classical ones on compatible subsets of $E$. Note that a subset is called compatible if it is contained in another subset of $E$ forming a Boolean algebra (i.e., it is contained in a classical subsystem of $E$ ) [11.

Some quantum logics entail unique conditional probabilities, many others don't. The classical Boolean algebras and the Hilbert space quantum logic (consisting of the closed subspaces or, equivalently, the selfadjoint projection operators) do and, in the latter case, conditionalization becomes identical with the state transition of the Lüders - von Neumann (i.e., projective) quantum measurement process [30. Therefore, the quantum logics which possess unique conditional probabilities can be regarded as a generalized model of projective quantum measurement. Examples that are neither classical Boolean algebras nor Hilbert space quantum logics (nor sublogics of them) can be constructed using the exceptional real Jordan algebras [30, 31].

In this framework, a very special type of conditional probability emerges in the non-classical case 30, 31. It describes the probability for the transition from a past event $e$ to a future event $f$, independently of any underlying state, and results solely from the algebraic structure of the quantum logic $E$. This probability exists only for certain pairs $e$ and $f$ in $E$. It exists for all $f \in E$, if $e$ is minimal in $E$ (i.e., $e$ is an atom). The states resulting in this way are called atomic. They represent a generalization of the pure states in the usual quantum mechanical Hilbert space setting.

After the early pioneering work by Birkhoff and von Neumann in 1936 [10], quantum logics have been studied extensively between 1960 and 1995 [5, 9, 25], 26, 33, 34, 39, 40, 41. Various forms of conditional probability have also been considered [5, 13, 17, 19, 23, 24]. However, the quantum logics which possess unique conditional probabilities and particularly the special type of the stateindependent conditional probability had not received any attention before the author's work [29, 30, 31].

This special type of conditional probability is used in the present paper to study two well-known quantum key distribution protocols (including a variant of one of them). The first one was invented by Charles H. Bennett and Gilles Brassard in 1984 [7, based on earlier work in the 1970ies by Stephen Wiesner which was not published before 1983 [42]. Its variant is due to the first coinventor [6]. The second protocol was proposed by Artur K. Ekert in 1991 [18.

These quantum key distribution protocols are generalized in a two-fold way. First, they are transferred to the quantum logics possessing the conditional 
probability calculus; this shows that quantum key distribution does not necessarily require the full-blown Hilbert space formalism of quantum mechanics and works as well in a much more general and abstract model of the projective quantum measurement process. Second, system properties are used instead of states. Pure states, wavefunctions and their collapse are not needed.

Before turning to quantum key distribution, the no-cloning theorem of Ref. 32 is revisited and extended by treating the cloning of system properties, since only the cloning of atomic states was considered in Ref. [32.

Moreover, the special type of conditional probability mentioned above is identified as the quantum origin of the no-cloning theorem as well as of the superior security of the key distribution processes. Other generalizations of the no-cloning theorem 1, 2, and quantum key distribution 4, using the generalized probabilistic theories, do not cover the results presented here, since they do not capture this special type of conditional probability.

The paper is organized as follows. The next two sections restate some material from Refs. [30, 31, 32 as far as needed in the present paper. In section 2 , the algebraic structure of a quantum logic is outlined and, in section 3 , the conditional probability calculus. In section 4 , the extension of the no-cloning theorem is introduced. The generalized versions of the quantum key distribution protocols are presented in section 5 .

\section{Compatibility in orthomodular partially ordered sets}

In quantum mechanics, the measurable quantities of a physical system are represented by observables. Most simple are those observables where only the two values 'true' and 'false' (or ' 1 ' and ' 0 ') are possible as measurement outcome. They are elements of a mathematical structure called quantum logic, are usually called propositions, and they are called events in probabilistic approaches. The elements of the quantum logic can also be understood as potential properties of the system under consideration. If, for instance, this system is an electron, its spin along each spatial axis $\vec{x}$ can take one of the two values $+\hbar / 2$ and $-\hbar / 2$ and, for each $\vec{x}$, 'spin along $\vec{x}=+\hbar / 2$ ' and 'spin along $\vec{x}=-\hbar / 2$ ' represent two elements of the quantum logic.

In this paper, a quantum logic shall be an orthomodular partially ordered set $E$ with the partial ordering $\leq$, the orthocomplementation ', the smallest element 0 and the largest element $\mathbb{I}[5,9,25,34$. This means that the following conditions are satisfied by all $e, f \in E$ :

(A) $e \leq f$ implies $f^{\prime} \leq e^{\prime}$.

(B) $\left(e^{\prime}\right)^{\prime}=e$.

(C) $e \leq f^{\prime}$ implies $e \vee f$, the supremum of $e$ and $f$, exists.

(D) $e \vee e^{\prime}=\mathbb{I}$.

(E) $f \leq e$ implies $e=f \vee\left(e \wedge f^{\prime}\right)$.

(orthomodular law) 
Here, $e \wedge f$ denotes the infimum of $e$ and $f$, which exists if and only if $e^{\prime} \vee f^{\prime}$ exists. Two elements $e, f \in E$ are called orthogonal if $e \leq f^{\prime}$ or, equivalently, $f \leq e^{\prime}$. An element $e \neq 0$ in $E$ is called an atom if there is no element $f$ in $E$ with $f \leq e$ and $0 \neq f \neq e$.

The interpretation of this mathematical terminology is as follows: two orthogonal elements represent mutually exclusive events, propositions or system properties; $e^{\prime}$ is the negation of $e$, and $e \vee f$ is the disjunction of the two exclusive elements $e$ and $f$.

It is not assumed that $E$ is a lattice (in a lattice, there is a smallest upper bound $e \vee f$ and largest lower bound $e \wedge f$ for any two elements $e$ and $f$ ). If $E$ were a lattice and satisfied the distributivity law

$$
e \wedge(f \vee g)=(e \wedge f) \vee(e \wedge g) \text { for all } e, f, g \in E,
$$

$E$ would become a Boolean lattice or Boolean algebra. The orthomodular law is a weakening of the distributivity law.

Classical probability theory uses Boolean algebras as mathematical structure for the random events, and it can be expected that those subsets of $E$, which are Boolean algebras, behave classically. Therefore, a subset $E_{0}$ of $E$ is called compatible if there is a Boolean algebra $B$ with $E_{0} \subseteq B \subseteq E$. Two elements $e$ and $f$ in $E$ are called compatible, if $\{e, f\}$ forms a compatible subset. Note that the supremum $e \vee f$ and the infimum $e \wedge f$ exist for any compatible pair $e$ and $f$ in $E$ and that the distributivity law holds in any compatible subset of $E$. Any subset with pairwise orthogonal elements is compatible [11.

Two subsets $E_{1}$ and $E_{2}$ of $E$ are called compatible with each other if the union of any compatible subset of $E_{1}$ with any compatible subset of $E_{2}$ is a compatible subset of $E$. Note that this does not imply that $E_{1}$ or $E_{2}$ themselves are compatible subsets.

A subset of an orthomodular lattice is compatible if each pair of elements in this subset is compatible. However, the pairwise compatibility of the elements of a subset of an orthomodular partially ordered set does not any more imply the compatibility of this subset [11.

A quantum logical structure, which is more general than the orthomodular partially ordered sets, has been used in Refs. [30, 31. This more general structure is sufficient when only compatible pairs of elements in the quantum logic are considered. However, compatible subsets with more than two elements will play an important role in this paper.

A quantum logic is a purely algebraic structure, unfurling its full potential only when its state space has some nice properties which shall be considered in the next section. 


\section{Non-classical conditional probability}

The states on the orthomodular partially ordered set $E$ are the analogue of the probability measures in classical probability theory, and conditional probabilities can be defined similar to their classical prototype.

A state $\rho$ allocates the probability $\rho(f)$ with $0 \leq \rho(f) \leq 1$ to each element $f \in E$, is additive for orthogonal elements, and $\rho(\mathbb{I})=1$. It then follows that $\rho(f) \leq \rho(e)$ for any two elements $e, f \in E$ with $f \leq e$.

The conditional probability of an element $f$ under another element $e$ is the updated probability for $f \in E$ after the outcome of a first measurement has been $e \in E$; it is denoted by $\rho(f \mid e)$. Mathematically, it is defined by the conditions that the map $E \ni f \rightarrow \rho(f \mid e)$ is a state on $E$ and that it coincides with the classical conditional probability for those $f$ which are compatible with $e$; this means

$$
\rho(f \mid e)=\frac{\rho(e \wedge f)}{\rho(e)},
$$

if $f$ is compatible with $e$. It must be assumed that $\rho(e) \neq 0$.

However, among the orthomodular partially ordered sets, there are many where no states or no conditional probabilities exist, or where the conditional probabilities are ambiguous. It shall now be assumed for the remaining part of this paper that

(F) there is a state $\rho$ on $E$ with $\rho(e) \neq 0$ for each $e \in E$ with $e \neq 0$,

(G) $E$ possesses unique conditional probabilities, and

(H) the state space of $E$ is strong; i.e., if

$$
\{\rho \mid \rho \text { is a state with } \rho(f)=1\} \subseteq\{\rho \mid \rho \text { is a state with } \rho(e)=1\}
$$

holds for $e, f \in E$, then $f \leq e$.

If $\rho$ is a state with $\rho(e)=1$ for some element $e \in E$, then $\rho(f \mid e)=\rho(f)$ for all $f \in E$. This follows from $(\mathrm{G})$.

For some pairs $e$ and $f$ in $E$, the conditional probability does not depend on the underlying state; this means $\rho_{1}(f \mid e)=\rho_{2}(f \mid e)$ for all states $\rho_{1}$ and $\rho_{2}$ with $\rho_{1}(e) \neq 0 \neq \rho_{2}(e)$. This special conditional probability is then denoted by $\mathbb{P}(f \mid e)$. The following two conditions are equivalent for any pair $e, f \in E$ :

(i) $\mathbb{P}(f \mid e)$ exists and $\mathbb{P}(f \mid e)=s$.

(ii) $\rho(e)=1$ implies $\rho(f)=s$ for the states $\rho$ on $E$.

Due to condition (H), $f \leq e$ holds for two elements $e$ and $f$ in $E$ if and only if $\mathbb{P}(e \mid f)=1$. Moreover, $e$ and $f$ are orthogonal if and only if $\mathbb{P}(e \mid f)=0$.

$\mathbb{P}(f \mid e)$ exists for all $f \in E$ if and only if $e$ is an atom (minimal element in $E)$, which results in the atomic state $\mathbb{P}_{e}$ defined by $\mathbb{P}_{e}(f):=\mathbb{P}(f \mid e)$. This is the unique state allocating the probability value 1 to the atom $e$. 
Quantum mechanics uses a special quantum logic; it consists of the selfadjoint projection operators on a Hilbert space $H$ and is an orthomodular lattice. Compatibility here means that the self-adjoint projection operators commute. Conditions $(\mathrm{F})$ and $(\mathrm{H})$ are satisfied, and the unique conditional probabilities exist (G); it has been shown in Ref. [30] that, with two self-adjoint projection operators $e$ and $f$ on $H$, the conditional probability has the shape

$$
\rho(f \mid e)=\frac{\operatorname{trace}(\text { aefe })}{\operatorname{trace}(a e)}=\frac{\operatorname{trace}(\text { eaef })}{\operatorname{trace}(a e)}
$$

for a state $\rho$ defined by the statistical operator $a$ (i.e., $a$ is a self-adjoint operator on $H$ with non-negative spectrum and $\operatorname{trace}(a)=1)$. The above identity reveals that conditionalization becomes identical with the state transition of the Lüders - von Neumann measurement process. Therefore, the conditional probabilities can be regarded as a generalized mathematical model of projective quantum measurement.

$\mathbb{P}(f \mid e)$ exists with $\mathbb{P}(f \mid e)=s$ if and only if the operators $e$ and $f$ on $H$ satisfy the algebraic identity efe $=s e$. This transition probability between the outcomes of two consecutive measurements is independent of any underlying state and results from the algebraic structure of the quantum logic.

The atoms are the self-adjoint projections on the one-dimensional subspaces of $H$; if $e$ is an atom and $|\xi\rangle$ a normalized vector in the corresponding onedimensional subspace, then $\mathbb{P}(f \mid e)=\langle\xi \mid f \xi\rangle$. The atomic states thus coincide with the quantum mechanical pure states or vector states. Their general nonorthogonality is quite characteristic of quantum mechanics. If $f$ is an atom as well and $|\eta\rangle$ a normalized vector in the corresponding one-dimensional subspace, then $\mathbb{P}(f \mid e)=|\langle\eta \mid \xi\rangle|^{2}$.

Now it becomes evident why $|\langle\eta \mid \xi\rangle|^{2}$ represents a probability. In the usual quantum mechanical setting, this probabilistic interpretation (Born rule) is a later add-on to the Hilbert space model, enforced by experimental evidence, but rather unmotivated from the theoretical point of view.

In the case of the electron spin, with a fixed spatial axis $\vec{x}$, 'spin along $\vec{x}=$ $+\hbar / 2$ ' and 'spin along $\vec{x}=-\hbar / 2$ ' represent two elements of the quantum logic which are orthogonal and compatible. With two different axes $\vec{x}$ and $\vec{y}$, however, 'spin along $\vec{x}=+\hbar / 2$ ' and 'spin along $\vec{y}=+\hbar / 2$ ' represent two elements of the quantum logic which are not compatible. The probability

$$
\mathbb{P} \text { ('spin along } \vec{x}=+\hbar / 2 \text { '|'spin along } \vec{y}=+\hbar / 2 \text { ') }
$$

exists; it depends on the angle $\theta$ between the two axes and equals $\cos ^{2}(\theta / 2)$ $(=1 / 2$ for $\theta=\pi / 2)$. 


\section{The generalized no-cloning theorem}

In the remaining sections of this paper, a quantum logic shall always be an orthomodular partially ordered set satisfying $(\mathrm{F}),(\mathrm{G})$ and $(\mathrm{H})$. This means that the existence of unique conditional probabilities is always assumed.

In the following, two lemmas from Ref. 32] will be needed. The first one concerns the invariance of $\mathbb{P}(\mid)$ under morphisms and the second one the multiplicativity of $\mathbb{P}(\mid)$ under certain conditions. These lemmas shall be restated here without their proofs which can be found in Ref. 32.

With two quantum logics $E$ and $F$, a morphism is a map $T: E \rightarrow F$ satisfying the following three conditions: (i) $T e_{1} \leq T e_{2}$ for $e_{1}, e_{2} \in E$ with $e_{1} \leq e_{2}$, (ii) $T\left(e^{\prime}\right)=(T e)^{\prime}$ for all $e \in E$, and (iii) $T \mathbb{I}=\mathbb{I}$.

Lemma 1: Suppose $E$ and $F$ are quantum logics and $T: E \rightarrow F$ is a morphism. If $\mathbb{P}\left(e_{2} \mid e_{1}\right)$ exists for two elements $e_{1}$ and $e_{2}$ in $E$ with $T e_{1} \neq 0$, then $\mathbb{P}\left(T e_{2} \mid T e_{1}\right)$ exists and $\mathbb{P}\left(T e_{2} \mid T e_{1}\right)=\mathbb{P}\left(e_{2} \mid e_{1}\right)$.

Suppose that $E$ is a quantum logic and that two compatible copies of it are contained in the larger quantum logic $L$. This means that there are two injective morphisms $\pi_{a}: E \rightarrow L$ and $\pi_{b}: E \rightarrow L$ and that the subsets $\pi_{a}(E)$ and $\pi_{b}(E)$ of $L$ are compatible with each other.

Lemma 2: If $\mathbb{P}\left(e_{2} \mid e_{1}\right)$ and $\mathbb{P}\left(f_{2} \mid f_{1}\right)$ both exist for $e_{1}, e_{2}, f_{1}, f_{2} \in E$, then

$$
\mathbb{P}\left(\left(\pi_{a} e_{2}\right) \wedge\left(\pi_{b} f_{2}\right) \mid\left(\pi_{a} e_{1}\right) \wedge\left(\pi_{b} f_{1}\right)\right)
$$

exists and

$$
\mathbb{P}\left(\left(\pi_{a} e_{2}\right) \wedge\left(\pi_{b} f_{2}\right) \mid\left(\pi_{a} e_{1}\right) \wedge\left(\pi_{b} f_{1}\right)\right)=\mathbb{P}\left(e_{2} \mid e_{1}\right) \mathbb{P}\left(f_{2} \mid f_{1}\right) .
$$

In the usual quantum mechanical setting, the cloning is performed by a unitary transformation on the Hilbert space tensor product. In this paper, it shall be performed by an automorphism of $L$ containing the two compatible copies of $E$.

Theorem 1: Suppose that $e_{1}, e_{2}, f$ are elements of the quantum logic $E$, that $\mathbb{P}\left(e_{2} \mid e_{1}\right)$ exists and that $T: L \rightarrow L$ is an automorphism with

$$
T\left(\left(\pi_{a} e_{k}\right) \wedge\left(\pi_{b} f\right)\right)=\left(\pi_{a} e_{k}\right) \wedge\left(\pi_{b} e_{k}\right)
$$

for $k=1,2$. Then $\mathbb{P}\left(e_{2} \mid e_{1}\right) \in\{0,1\}$; this means that either $e_{1}$ and $e_{2}$ are orthogonal or $e_{1} \leq e_{2}$. 
Proof. By Lemma 1 and 2, on the one hand,

$$
\begin{aligned}
\mathbb{P}\left(T \left(\left(\pi_{a} e_{2}\right)\right.\right. & \left.\left.\wedge\left(\pi_{b} f\right)\right) \mid T\left(\left(\pi_{a} e_{1}\right) \wedge\left(\pi_{b} f\right)\right)\right) \\
= & \mathbb{P}\left(\left(\pi_{a} e_{2}\right) \wedge\left(\pi_{b} e_{2}\right) \mid\left(\pi_{a} e_{1}\right) \wedge\left(\pi_{b} e_{1}\right)\right) \\
= & \mathbb{P}\left(\pi_{a} e_{2} \mid \pi_{a} e_{1}\right) \mathbb{P}\left(\pi_{b} e_{2} \mid \pi_{b} e_{1}\right) \\
= & \left(\mathbb{P}\left(e_{2} \mid e_{1}\right)\right)^{2}
\end{aligned}
$$

and, on the other hand,

$$
\begin{aligned}
\mathbb{P}\left(T \left(\left(\pi_{a} e_{2}\right)\right.\right. & \left.\left.\wedge\left(\pi_{b} f\right)\right) \mid T\left(\left(\pi_{a} e_{1}\right) \wedge\left(\pi_{b} f\right)\right)\right) \\
= & \mathbb{P}\left(\left(\pi_{a} e_{2}\right) \wedge\left(\pi_{b} f\right) \mid\left(\pi_{a} e_{1}\right) \wedge\left(\pi_{b} f\right)\right) \\
= & \mathbb{P}\left(\pi_{a} e_{2} \mid \pi_{a} e_{1}\right) \mathbb{P}\left(\pi_{b} f \mid \pi_{b} f\right) \\
= & \mathbb{P}\left(e_{2} \mid e_{1}\right) .
\end{aligned}
$$

Thus, $\left(\mathbb{P}\left(e_{2} \mid e_{1}\right)\right)^{2}=\mathbb{P}\left(e_{2} \mid e_{1}\right)$ and therefore $\mathbb{P}\left(e_{2} \mid e_{1}\right) \in\{0,1\}$.

Theorem 1 means that the cloning or copying of the two unknown system properties $e_{1}, e_{2}$ becomes impossible, when $\mathbb{P}\left(e_{2} \mid e_{1}\right)$ exists and when this probability is different from 0 and 1 . If the system property is unknown in a larger set with more than two elements, the cloning is impossible whenever this probability exists and is different from 0 and 1 for any pair of elements in this set.

$\mathbb{P}\left(e_{2} \mid e_{1}\right)$ always exists for atoms $e_{1}, e_{2}$ and, in this case, cloning is possible only if the atoms are orthogonal or identical. Atoms correspond to the atomic states (the generalization of the quantum mechanical pure states), and the cloning of such atomic states was studied in Ref. 32] which, however, required some additional assumptions that are not needed here.

Theorem 1 is substantially more general than the result in Ref. [32] and than the well-known quantum mechanical no-cloning theorem. Even in the usual Hilbert space setting, Theorem 1 covers cases where $e_{1}$ and $e_{2}$ are not atomic and these cases are not included in other results. This becomes possible by considering the cloning of system properties instead of states and by using the specific probability $\mathbb{P}(\mid)$.

The original quantum mechanical no-cloning theorem [43, 16, has been extended into different other directions: to mixed states [3], to $\mathrm{C}^{*}$-algebras [15], to finite-dimensional generic probabilistic models [1, 2], and to universal cloning 28]. Possible is only the approximate or imperfect cloning [14, 12, 27]. However, none of these extensions covers the above result. 


\section{Quantum key distribution}

\subsection{The first protocol}

The quantum key distribution protocol invented by Charles H. Bennett and Gilles Brassard in 1984 [7] shall be considered first, but instead of using the ordinary quantum mechanical Hilbert space formalism and pure states, it will be transferred to the more general setting described in the last three sections.

Assume that two parties, usually named Alice and Bob, use a system, the properties of which form a quantum logic $E$ containing two elements $e$ and $f$ with

$$
\mathbb{P}(e \mid f)=\mathbb{P}\left(e \mid f^{\prime}\right)=\mathbb{P}(f \mid e)=\mathbb{P}\left(f \mid e^{\prime}\right)=1 / 2 .
$$

Both agree in advance on using this system and these properties $e$ and $f$. Alice encodes the bit 1 in either $e$ or $f$ and the bit 0 in either $e^{\prime}$ or $f^{\prime}$. She decides at random whether she uses $e, e^{\prime}$ or $f, f^{\prime}$ for the bit. She then sends the system carrying the encoded bit to Bob. Theorem 1 ensures that an eavesdropper cannot make use of copying.

Bob does not know whether Alice used $e, e^{\prime}$ or $f, f^{\prime}$ and decides at random whether he measures $e$ or $f$. In the case he measures $e$, the outcome $e$ means the bit 1 and the outcome $e^{\prime}$ means the bit 0 . In the case he measures $f$, the outcome $f$ means the bit 1 and the outcome $f^{\prime}$ means the bit 0 . When using the same property as Alice, he gets the right bit with probability 1 since $\mathbb{P}(e \mid e)=\mathbb{P}(f \mid f)=1$ and $\mathbb{P}\left(e \mid e^{\prime}\right)=\mathbb{P}\left(f \mid f^{\prime}\right)=0$; when using the other property he gets a useless random bit, since $\mathbb{P}(f \mid e)=\mathbb{P}\left(f \mid e^{\prime}\right)=\mathbb{P}(e \mid f)=\mathbb{P}\left(e \mid f^{\prime}\right)=1 / 2$.

After Bob's measurement, he uses any classical and possibly public communication channel to announce to Alice whether he has measured $e$ or $f$, but he does not tell the result he obtained. Over the same communication channel, Alice then only announces whether or not they both used the same one of the two system properties. If they used the same one, they keep the bit; if not,they disregard it. Each of these two cases occurs with probability $1 / 2$.

After repeating the above procedure sufficiently many times with equal systems, Alice and Bob get a bit string which can they then use as key for the encryption of messages which are then sent over the classical communication channel.

Due to the no-cloning theorem in section 4, an eavesdropper cannot generate a copy of the signal which Alice sends to Bob without destroying the signal which Bob receives. In this case, Bob would get the wrong bits (also when using the same property $e$ or $f$ as Alice) and the key would not work, since Alice and Bob use different bit strings. The eavesdropper can thus hinder the key distribution, but does not have access to the key without being detected.

When the key works, Alice and Bob can be sure that nobody has a copy of the key. They can check this by exchanging some unimportant test messages encrypted with the key over the classical channel, before starting to exchange secret information. Another possibility is that they compare a small, but sufficiently large subset of their bit strings; in the case of perfect coincidence, they 
can be sure that nobody has a copy of the key. If they cannot be sure, they discard the key and repeat the whole procedure to generate a new key.

Other and perhaps better procedures for this classical post-processing have been proposed 17, 18, 20, 36, 37, but are not relevant for the scope of this paper.

\subsection{A variant of the first protocol}

Some time later, one of the co-inventors of the first protocol detected that it sufficient for Alice to use only the two non-compatible quantum properties $e$ and $f$ instead of all the four $e, e^{\prime}, f, f^{\prime}$ and introduced the following variant [6].

Under the same assumptions as with the first protocol in subsection 5.1, Alice uses $e$ for encoding the bit 1 and $f$ for encoding the bit 0 ; she never uses $e^{\prime}$ and $f^{\prime}$.

Bob again decides at random whether he measures $e$ or $f$. In the case he measures $e$, the outcome $e$ means the bit 1 and, in the case he measures $f$, the outcome $f$ means the bit 0 . The other measurement outcomes $e^{\prime}$ and $f^{\prime}$ are useless and result from two different reasons; either Bob's random selection among $e$ and $f$ does not coincide with Alice's coding or the transmission was not correct because of the presence of an eavesdropper or some transmission problems.

The procedure is then continued in the same way as described in the last subsection.

\subsection{The second protocol}

A further quantum key distribution protocol invented by Artur K. Ekert in 1991 [18] shall now be considered. The major difference to the first one is that it uses two entangled subsystems for the transmission of each bit. Alice and Bob can exchange these subsystems in advance, before Alice encodes the bit. After this, no system is transmitted from Alice to Bob.

Consider a quantum logic $E$ with five elements $e_{a}, f_{a}, e_{b}, f_{b}, d$ satisfying the following three conditions:

(i) The subsets $\left\{e_{a}, e_{a}^{\prime}, f_{a}, f_{a}^{\prime}\right\}$ and $\left\{e_{b}, e_{b}^{\prime}, f_{b}, f_{b}^{\prime}\right\}$ are compatible with each other.

(ii) $\mathbb{P}\left(e_{k} \mid f_{k}\right)=\mathbb{P}\left(e_{k} \mid f_{k}^{\prime}\right)=\mathbb{P}\left(f_{k} \mid e_{k}\right)=\mathbb{P}\left(f_{k} \mid e_{k}^{\prime}\right)=1 / 2$ for $k=a, b$.

(iii) $\mathbb{P}\left(e_{k} \mid d\right)=\mathbb{P}\left(f_{k} \mid d\right)=1 / 2$ for $k=a, b$ and $\mathbb{P}\left(e_{a} \wedge e_{b} \mid d\right)=\mathbb{P}\left(f_{a} \wedge f_{b} \mid d\right)=1 / 2$, $\mathbb{P}\left(e_{a} \wedge f_{b} \mid d\right)=\mathbb{P}\left(f_{a} \wedge e_{b} \mid d\right)=1 / 4$.

Note that (iii) implies some further identities. From $\mathbb{P}\left(e_{a} \mid d\right)=\mathbb{P}\left(e_{a} \wedge e_{b} \mid d\right)+$ $\mathbb{P}\left(e_{a} \wedge e_{b}^{\prime} \mid d\right)$, it follows that $\mathbb{P}\left(e_{a} \wedge e_{b}^{\prime} \mid d\right)=0$. The identities $\mathbb{P}\left(e_{a}^{\prime} \wedge e_{b} \mid d\right)=0$, $\mathbb{P}\left(f_{a} \wedge f_{b}^{\prime} \mid d\right)=0$ and $\mathbb{P}\left(f_{a}^{\prime} \wedge f_{b} \mid d\right)=0$ follow in the same way. The orthogonal decomposition $\mathbb{I}=\left(e_{a} \wedge e_{b}\right) \vee\left(e_{a} \wedge e_{b}^{\prime}\right) \vee\left(e_{a}^{\prime} \wedge e_{b}\right) \vee\left(e_{a}^{\prime} \wedge e_{b}^{\prime}\right)$ then implies

$$
\mathbb{P}\left(e_{a}^{\prime} \wedge e_{b}^{\prime} \mid d\right)=1 / 2
$$


and

$$
\mathbb{P}\left(f_{a}^{\prime} \wedge f_{b}^{\prime} \mid d\right)=1 / 2
$$

follows in the same way. Moreover, $\mathbb{P}\left(e_{a} \mid d\right)=\mathbb{P}\left(e_{a} \wedge f_{b} \mid d\right)+\mathbb{P}\left(e_{a} \wedge f_{b}^{\prime} \mid d\right)$ implies

$$
\mathbb{P}\left(e_{a} \wedge f_{b}^{\prime} \mid d\right)=1 / 4 \text {. }
$$

In the same way, it is concluded that

$$
1 / 4=\mathbb{P}\left(e_{a}^{\prime} \wedge f_{b} \mid d\right)=\mathbb{P}\left(e_{a}^{\prime} \wedge f_{b}^{\prime} \mid d\right)=\mathbb{P}\left(f_{a} \wedge e_{b}^{\prime} \mid d\right)=\mathbb{P}\left(f_{a}^{\prime} \wedge e_{b} \mid d\right)=\mathbb{P}\left(f_{a}^{\prime} \wedge e_{b}^{\prime} \mid d\right) .
$$

In the beginning of the key distribution process, the system is prepared in such a way that property $d$ holds. The subsets $\left\{e_{a}, e_{a}^{\prime}, f_{a}, f_{a}^{\prime}\right\}$ and $\left\{e_{b}, e_{b}^{\prime}, f_{b}, f_{b}^{\prime}\right\}$ represent two subsystems; the first one is Alice's and the second one is Bob's.

The further proceeding is similar to the first quantum key distribution protocol. The only difference is that Alice and Bob use their different subsystems instead of transmitting the system from Alice to Bob.

However, it is more difficult to see that Bob gets the same bit as Alice when she uses $e_{a}, e_{a}^{\prime}$ and he $e_{b}, e_{b}^{\prime}$ or when she uses $f_{a}, f_{a}^{\prime}$ and he $f_{b}, e_{b}^{\prime}$. Assume that Alice encodes the bit 1 using $e_{a}$ and that Bob measures $e_{b}$. Since $e_{a}$ and $e_{b}$ are compatible, the conditional probability that Bob gets $e_{b}$ after Alice got $e_{a}$ is

$$
\frac{\mathbb{P}\left(e_{a} \wedge e_{b} \mid d\right)}{\mathbb{P}\left(e_{a} \mid d\right)}
$$

and equals 1 because of condition (iii). The same holds for the pairs $e_{a}^{\prime}$ and $e_{b}^{\prime}$, $f_{a}$ and $f_{b}, f_{a}^{\prime}$ and $f_{b}^{\prime}$, using condition (iii) and the identities following from it. This means that Bob gets the right bit, when his random choice between $e$ and $f$ coincides with Alice's choice.

When their choices don't match, he gets a useless random bit, 0 or 1 , each with equal probability $1 / 2$. Assume that Alice encodes the bit 1 using $e_{a}$ and that Bob measures $f_{b}$. Then

$$
\frac{\mathbb{P}\left(e_{a} \wedge f_{b} \mid d\right)}{\mathbb{P}\left(e_{a} \mid d\right)}
$$

equals $1 / 2$ because of condition (iii). The same holds for the pairs $e_{a}$ and $f_{b}^{\prime}, e_{a}^{\prime}$ and $f_{b}, e_{a}^{\prime}$ and $f_{b}^{\prime}, f_{a}$ and $e_{b}, f_{a}$ and $e_{b}^{\prime}, f_{a}^{\prime}$ and $e_{b}, f_{a}^{\prime}$ and $e_{b}^{\prime}$, using condition (iii) and the identities following from it.

The key distribution procedure is continued in the same way as described in subsection 5.1 for the first protocol.

\subsection{The usual quantum mechanical Hilbert space model}

The quantum key distribution protocols shall now be studied in the special quantum logic consisting of the self-adjoint projection operators on a Hilbert space $H$. A reader familiar with the protocols will now recognize them if not yet in subsections 5.1, 5.2 and 5.3.

Consider a 2-dimensional Hilbert space $H$ with an orthonormal basis $\left|\xi_{1}\right\rangle$ and $\left|\xi_{2}\right\rangle$. A second orthonormal basis is then given by 


$$
\left|\eta_{1}\right\rangle:=\frac{1}{\sqrt{2}}\left(\left|\xi_{1}\right\rangle+\left|\xi_{2}\right\rangle\right) \text { and }\left|\eta_{2}\right\rangle:=\frac{1}{\sqrt{2}}\left(\left|\xi_{1}\right\rangle-\left|\xi_{2}\right\rangle\right) .
$$

Now define the following self-adjoint projection operators:

$$
e:=\left|\eta_{1}\right\rangle\left\langle\eta_{1}\right| \text { and } f:=\left|\xi_{1}\right\rangle\left\langle\xi_{1}\right| .
$$

They satisfy the assumptions needed for the first protocol and its variant:

$$
\mathbb{P}(e \mid f)=\mathbb{P}\left(e \mid f^{\prime}\right)=\mathbb{P}(f \mid e)=\mathbb{P}\left(f \mid e^{\prime}\right)=1 / 2 .
$$

Furthermore, it here becomes evident that the situation, where $\mathbb{P}(\mid)$ exists with $0 \neq \mathbb{P}(\mid) \neq 1$, is an extension of the superposition principle of Hilbert space quantum mechanics to a much more general setting.

For the second protocol, consider the tensor product $H \otimes H$, its element

$$
|\psi\rangle:=\frac{1}{\sqrt{2}}\left(\left|\xi_{1}, \xi_{1}\right\rangle+\left|\xi_{2}, \xi_{2}\right\rangle\right) \in H \otimes H
$$

and define

$$
\begin{gathered}
d:=|\psi\rangle\langle\psi|, \\
e_{a}:=e \otimes \mathbb{I}, f_{a}:=f \otimes \mathbb{I}, \\
e_{b}:=\mathbb{I} \otimes e, f_{b}:=\mathbb{I} \otimes f,
\end{gathered}
$$

where $\mathbb{I}$ is the identity operator on $H$. They satisfy the conditions (i), (ii) and (iii), needed for the second protocol.

The element $|\psi\rangle$ in the tensor product of the two Hilbert spaces represents a typical entangled state. A different way of thinking, suggested by the approach presented in this paper, is to attribute the entanglement not to states, but to system properties and, in this special case, to the element $d=|\psi\rangle\langle\psi|$ of the quantum logic; it represents a property of the total system which cannot be described by the properties of the two individual subsystems.

In the physics literature, the elements of the Hilbert space $H$ are often called wavefunctions. They don't have any equivalent in the general and abstract setting provided by the quantum logics possessing a conditional probability calculus. Even in usual quantum mechanical setting, the key distribution protocols (and the no-cloning theorem) can do without the wavefunctions; instead, the elements of the Hilbert space quantum logic (the self-adjoint projection operators) together with the special state-independent conditional probability $\mathbb{P}(\mid)$ can be used. This probability, representing the transition probability between the measurement outcomes, then replaces the measurement induced so-called collapse of the wavefunction and becomes the quantum origin of the no-cloning theorem as well as of the superior security of the key distribution protocols. 


\section{Conclusion}

In this paper, it has been shown that the quantum no-cloning theorem and quantum key distribution allow a much more general and abstract access than commonly thought. Instead of the usual quantum mechanical Hilbert space model, a general non-classical extension of conditional probability has been used, which includes the usual model as a special case.

Equally important may be that, even in usual quantum mechanics, more cases are covered, since any elements in the quantum logic and not only the atoms (which correspond to the pure states) can be used for quantum key distribution. This then includes the quantum logics formed by the self-adjoint idempotent elements of the type II or type III von Neumann factors which occur naturally in relativistic quantum field theory and in quantum statistical mechanics of infinite systems [44, 35. These quantum logics do not contain any atom and do not possess any normal pure state, but there are elements $e$ and $f$ with $0<\mathbb{P}(f \mid e)<1$.

This special type of conditional probability $\mathbb{P}(f \mid e)$ could be identified as the quantum origin of the no-cloning theorem and the superior security of the key distribution protocols considered. The probability $\mathbb{P}(f \mid e)$ is a transition probability between the two events, propositions or system properties $e$ and $f$. It depends only on $e$ and $f$ and does not depend on any underlying quantum state. It results solely from the algebraic structure of the quantum logic.

This means that the usual assumption that every quantum system (e.g., a microphysical particle or the whole universe) is in a quantum state is not necessary any more to understand the probabilities observed in the quantum physical realm. No wavefunction and no collapse of it is needed. Some interpretational problems with quantum mechanics, resulting from this usual assumption, might thus be removed or shifted to another kind of questions. So far, the special type of conditional probability $\mathbb{P}(\mid)$ has not attracted much attention, although it

may have more profound implications for the foundations and interpretation of quantum theory, quantum information theory and the philosophical question, what actually constitutes physical reality.

Only the two oldest and best-known quantum key distribution protocols (including a variant) have been considered in the present paper, although there are more [20, 37. Moreover, quantum key distribution is only one of several tasks in quantum information theory. Others are entanglement-assisted quantum teleportation [8], quantum computing and specific quantum algorithms like Grover's search algorithm 21, 22 or Shor's algorithm for integer factorization 38. The question, whether these tasks allow the same general and abstract access as the quantum key distribution protocols considered here, will be tackled in a forthcoming paper. The essential feature needed for all of them is quantum entanglement and, that this feature can be transferred to the general and abstract setting, has already been demonstrated successfully in the treatment of the second quantum distribution protocol. A new way of thinking required here is to attribute the entanglement not to states, but to system properties. 


\section{References}

[1] H. Barnum, J. Barrett, M. Leifer, and A. Wilce. Cloning and broadcasting in generic probabilistic theories. arXiv:quant-ph/0611295, 2006.

[2] H. Barnum, J. Barrett, M. Leifer, and A. Wilce. Generalized nobroadcasting theorem. Physical Review Letters, 99(24):240501, 2007.

[3] H. Barnum, C. M. Caves, C. A. Fuchs, R. Jozsa, and B. Schumacher. Noncommuting mixed states cannot be broadcast. Physical Review Letters, 76(15):2818, 1996.

[4] J. Barrett. Information processing in generalized probabilistic theories. Physical Review A, 75(3):032304, 2007.

[5] E. G. Beltrametti, G. Cassinelli, and G.-C. Rota. The logic of quantum mechanics. Cambridge University Press, 1984.

[6] C. H. Bennett. Quantum cryptography using any two nonorthogonal states. Physical Review Letters, 68(21):3121, 1992.

[7] C. H. Bennett and G. Brassard. Quantum cryptography: Public key distribution and coin tossing. In Proceedings of IEEE International Conference on Computers, Systems and Signal Processing (Bangalore, India, Dec. 1984), volume 175, page 8, 1984.

[8] C. H. Bennett, G. Brassard, C. Crépeau, R. Jozsa, A. Peres, and W. K. Wootters. Teleporting an unknown quantum state via dual classical and Einstein-Podolsky-Rosen channels. Physical Review Letters, 70(13):1895, 1993.

[9] L. Beran. Orthomodular lattices. Springer, 1985.

[10] G. Birkhoff and J. von Neumann. The logic of quantum mechanics. Annals of Mathematics, 37:823-843, 1936.

[11] J. Brabec. Compatibility in orthomodular posets. Časopis pro pěstování matematiky, 104(2):149-153, 1979.

[12] D. Bruß, D. P. DiVincenzo, A. Ekert, C. A. Fuchs, C. Macchiavello, and J. A. Smolin. Optimal universal and state-dependent quantum cloning. Phys. Rev. A, 57:2368-2378, Apr 1998.

[13] J. Bub. Von Neumann's projection postulate as a probability conditionalization rule in quantum mechanics. Journal of Philosophical Logic, 6(1):381-390, 1977.

[14] V. Bužek and M. Hillery. Quantum copying: Beyond the no-cloning theorem. Physical Review A, 54(3):1844, 1996. 
[15] R. Clifton, J. Bub, and H. Halvorson. Characterizing quantum theory in terms of information-theoretic constraints. Foundations of Physics, 33(11):1561-1591, 2003.

[16] D. Dieks. Communication by EPR devices. Physics Letters A, 92(6):271$272,1982$.

[17] C. M. Edwards and G. T. Rüttimann. On conditional probability in GL spaces. Foundations of Physics, 20(7):859-872, 1990.

[18] A. K. Ekert. Quantum cryptography based on Bell's theorem. Phys. Rev. Lett., 67:661-663, Aug 1991.

[19] M. Friedman and H. Putnam. Quantum logic, conditional probability, and interference. Dialectica, 32(3-4):305-315, 1978.

[20] N. Gisin, G. Ribordy, W. Tittel, and H. Zbinden. Quantum cryptography. Reviews of Modern Physics, 74(1):145, 2002.

[21] L. K. Grover. A fast quantum mechanical algorithm for database search. In Proceedings of the twenty-eighth annual ACM symposium on Theory of computing, pages 212-219. ACM, 1996.

[22] L. K. Grover. Quantum mechanics helps in searching for a needle in a haystack. Physical Review Letters, 79(2):325, 1997.

[23] J. Gunson. On the algebraic structure of quantum mechanics. Communications in Mathematical Physics, 6(4):262-285, 1967.

[24] W. Guz. Conditional probability and the axiomatic structure of quantum mechanics. Fortschritte der Physik, 29(8):345-379, 1981.

[25] G. Kalmbach. Orthomodular lattices. Academic Press, London, 1983.

[26] H. A. Keller. Ein nicht-klassischer Hilbertscher Raum. Mathematische Zeitschrift, 172(1):41-49, 1980.

[27] Y. Kitajima. Imperfect cloning operations in algebraic quantum theory. Foundations of Physics, 45(1):62-74, 2015.

[28] T. Miyadera and H. Imai. No-cloning theorem on quantum logics. Journal of Mathematical Physics, 50(10):-, 2009.

[29] G. Niestegge. Statistische und deterministische vorhersagbarkeit bei der quantenphysikalischen messung. Helvetica Physica Acta, 71(2):163-183, 1998.

[30] G. Niestegge. Non-Boolean probabilities and quantum measurement. Journal of Physics A: Mathematical and General, 34(30):6031, 2001.

[31] G. Niestegge. An approach to quantum mechanics via conditional probabilities. Foundations of Physics, 38(3):241-256, 2008. 
[32] G. Niestegge. Non-classical conditional probability and the quantum nocloning theorem. Physica Scripta, 90(9):095101, 2015.

[33] C. Piron. Axiomatique quantique. Helvetica Physica Acta, 37(4-5):439-468, 1964.

[34] P. Pták and S. Pulmannová. Orthomodular structures as quantum logics. Kluwer, Dordrecht, 1991.

[35] S. Sakai. $C^{*}$-algebras and $W^{*}$-algebras. Springer Science \& Business Media, 2012.

[36] V. Scarani, A. Acin, G. Ribordy, and N. Gisin. Quantum cryptography protocols robust against photon number splitting attacks for weak laser pulse implementations. Physical Review Letters, 92(5):057901, 2004.

[37] V. Scarani, H. Bechmann-Pasquinucci, N. J. Cerf, M. Dušek, N. Lütkenhaus, and M. Peev. The security of practical quantum key distribution. Reviews of Modern Physics, 81(3):1301, 2009.

[38] P. W. Shor. Algorithms for quantum computation: Discrete logarithms and factoring. In Foundations of Computer Science, 1994 Proceedings., 35th Annual Symposium on, pages 124-134. IEEE, 1994.

[39] M. P. Soler. Characterization of Hilbert spaces by orthomodular spaces. Communications in Algebra, 23(1):219-243, 1995.

[40] V. S. Varadarajan. Geometry of Quantum Theory, Vol. 1. Van Nostrand Reinhold, New York, 1968.

[41] V. S. Varadarajan. Geometry of Quantum Theory, Vol. 2. Van Nostrand Reinhold, New York, 1970.

[42] S. Wiesner. Conjugate coding. ACM Sigact News, 15(1):78-88, 1983.

[43] W. K. Wootters and W. H. Zurek. A single quantum cannot be cloned. Nature, 299(5886):802-803, 1982.

[44] J. Yngvason. The role of type III factors in quantum field theory. Reports on Mathematical Physics, 55(1):135-147, 2005.

[45] H. P. Yuen. Amplification of quantum states and noiseless photon amplifiers. Physics Letters A, 113(8):405-407, 1986. 